\title{
Preliminary results of measuring occupational exposure to static magnetic fields at HVDC cables in Finland
}

\author{
J. Tonteri ${ }^{1}$, T. Heiskanen ${ }^{1}$, J. Elovaara ${ }^{1}$, M. Penttilä ${ }^{1}$ and L. Korpinen ${ }^{2, *}$ \\ ${ }^{1}$ Fingrid Oyj, Helsinki, Finland. \\ 2 The Clinical Physiology and Neurophysiology Unit, The North Karelia Central Hospital, Joensuu, Finland.
}

Received: 19 November 2016 / Accepted: 27 March 2017

\begin{abstract}
The aim of this study was to investigate the level of occupational exposure to static magnetic fields experienced by workers close to high voltage direct current (HVDC) cables in Finland. The measurements were conducted at a $500 \mathrm{kV}$ HVDC substation in Finland from 8 different measuring points with a commercial G100 handheld Gaussmeter by Coliy Technology. As a conclusion it can be stated that the static magnetic fields occurring in Fingrid's grid are safe for workers who do not have active implanted devices.
\end{abstract}

Keywords: static magnetic field / HVDC cable / occupational exposure

\section{Introduction}

During work tasks near high voltage direct current (HVDC) cables, workers are exposed to static electrical and static magnetic fields. Occupational exposure to static magnetic fields has been previously studied, for example, in Sweden (Feychting, 2005) where the studies mainly concentrated on long-term health effects like cancer, and in Italy (Bottauscio et al., 2009) where magnetic field emissions of a future underground HVDC cable were analyzed. However, there are only a few studies available of static magnetic field measurements in general, probably as the static magnetic flux densities can be easily calculated as well. Nevertheless, to raise the awareness of occupational exposure to static magnetic fields in such working conditions and to reduce possible fears of workers, it is reasonable to publish actual measurements as well.

The most extensive studies of the effects of static magnetic fields are Health Protection Agency (HPA's) static magnetic field report (HPA, 2008) and the International Commission on Non-ionizing Radiation Protection (ICNIRP) guidelines on limits of exposure to static magnetic fields, which also previously set the reference levels for safe static magnetic fields (ICNIRP, 2009). Occupational exposure to $50 \mathrm{~Hz}$ magnetic fields has been studied more broadly, for example, in Finland (Latva-Teikari et al., 2008; Korpinen and Pääkkönen, 2010; Korpinen et al., 2011).

In previous studies the occupational exposure to static magnetic fields near HVDC cables have been analyzed for example in Italy. The study shows that the calculated static magnetic fields and magnetic fields from harmonic frequencies of two future adjacent HVDC cables between Italy and

\footnotetext{
* Corresponding author: leenakorpinen@gmail.com
}

France under the Frejus highway were significantly lower than the effective ICNIRP reference levels. Calculations were made from different parts of the cable, where the configuration of the cables is also different. In addition different operating situations were taken into account. The used calculation method was a hybrid finite element - boundary element technique, based on the thin-shell formulation. The nominal current of a separate cable was $625 \mathrm{~A}$ while the highest nominal DC current in Finland is $1600 \mathrm{~A}$.

By the time of the Italian study the reference levels to magnetic fields were adopted by the 1999/519/EC Council Recommendation for the exposure of general public and by the Directive 2004/40/EC of the European Parliament for the exposure of workers. Nowadays, exposure limits have been updated by Directive 2013/35/EU (European Parliament and Council of the European Union, 2013).

The European Union governs the occupational exposure to static magnetic fields by Directive 2013/35/EU of the European Parliament and of the Council on the minimum health and safety requirements regarding the exposure of workers to the risks arising from physical agents (electromagnetic fields). It states that in a magnetic field from 0 to $1 \mathrm{~Hz}$, the exposure limit value (ELV) for sensory effects is $2 \mathrm{~T}$ as magnetic flux density in normal working conditions and $8 \mathrm{~T}$ for localized limbs. ELV for health effects in controlled working conditions is $8 \mathrm{~T}$. The action level (AL) for interference with active implanted devices, e.g., cardiac pacemakers, is $0.5 \mathrm{mT}$ and $3 \mathrm{mT}$ for attraction and projectile risk in the fringe field of high field strength sources $(>100 \mathrm{mT})$.

The aim of this study was to measure static magnetic flux densities at HVDC cables and cable terminals in Finland to determine the occupational exposure during work tasks. Especially, the goal was to ascertain if static magnetic fields exceed the ALs for interference with active implanted devices 
$(0.5 \mathrm{mT})$ and for attraction and projectile risk in the fringe field of high field strength sources $(3 \mathrm{mT})$ and the ELVs for sensory effects $(2 \mathrm{~T})$ and health effects $(8 \mathrm{~T})$. Measurements were made from Fenno-Skan 1 and Fenno-Skan 2 cables using a handheld Gaussmeter. Fenno-Skan cables are HVDC cables between Finland and Sweden.

\section{Selected measuring sites and methods}

The substation selected for this study was a $500 \mathrm{kV} \mathrm{DC}$ substation in Finland, where Fenno-Skan 1 and Fenno-Skan 2 HVDC cables are connected to HVDC overhead lines leading to converter stations. Converter stations were left out of the study, as no work tasks are performed inside the converter rooms with energized converters.

The selected substation has the highest nominal DC current in Finland and short distances between the live cables and workers, and was, therefore, selected as the worst case scenario site. It consists of Fenno-Skan 1 and Fenno-Skan 2 cable terminals, surge arresters, disconnectors, and HVDC overhead line couplings. The overhead lines are located $14 \mathrm{~m}$ from the ground. Nominal voltage for Fenno-Skan 1 is $400 \mathrm{kV}$, but recently $320 \mathrm{kV}$ and $500 \mathrm{kV}$ have been employed for FennoSkan 2. Nominal current for Fenno-Skan 1 is $1375 \mathrm{~A}$ and $1600 \mathrm{~A}$ for Fenno-Skan 2. In the Finnish transmission network, $1600 \mathrm{~A}$ is the highest nominal DC current; therefore, the static magnetic flux densities are also the highest as well as the occupational exposure to static magnetic fields. No significant AC currents flow in the DC cables as shown in Figures 1 and 2. The highest $\mathrm{AC}$ component is $8.5 \mathrm{~A}$ at around $300 \mathrm{~Hz}$, which is only $0.6 \%$ of the nominal DC current. Otherwise AC components are under $1 \mathrm{~A}$. Therefore, a very small amount of AC ripple passes the DC filters in Rauma converter station. Fenno-Skan 1 was constructed in 1989 and Fenno-Skan 2 in 2011.

Static magnetic flux densities of Fenno-Skan 1 and FennoSkan 2 cable terminals were measured from various distances close to ground level and over the underground Fenno-Skan 2 cable to define the occupational exposure to static magnetic fields while working near HVDC cables. Under the cable terminal, there is an unprotected gap that allows a worker to reach and touch the Fenno-Skan 2 cable. The first measurement was made by touching the cable with the measurement probe and thereafter from certain distances from the terminal. A commercial G100 hand-held Gaussmeter by Coliy Technology (7 Goethe Street, D-40237 Duesseldorf, Nordrhein-Westfalen, Germany) was used for measuring the magnetic flux density. Measurement probe was a standard Coliy 3-axis (Model Y08M150G1) probe. Typical accuracy of the instrument for DC fields is $0.8 \%$ and it can measure frequencies from DC to $30 \mathrm{kHz}$. Measurements were made hand-held and no tripod was used. The Fenno-Skan 2 cable terminal and the measurement procedure can be seen in Figure 3.

\section{Results}

The measurements were carried out in summer 2016. At the time of measurement, the current in the Fenno-Skan 2 cable was $1647 \mathrm{~A}$ and $1311 \mathrm{~A}$ in Fenno-Skan 1. Measurements were taken from different distances of Fenno-Skan 1 and FennoSkan 2 cable terminals and over the underground Fenno-Skan 2 cable as shown in Table 1.
Table 1 shows that the highest measured values were $2800 \mu \mathrm{T}$ for Fenno-Skan 2 and $1700 \mu \mathrm{T}$ for Fenno-Skan 1 cables. Over the underground Fenno-Skan 2 cable, the measured values were at most $200 \mu \mathrm{T}$. Results with distances closer than $1 \mathrm{~m}$ from Fenno-Skan 2 and $0.5 \mathrm{~m}$ from FennoSkan 1 cables inside the cable terminals were over the AL for interference with active implanted devices $(500 \mu \mathrm{T})$. All values were under the $\mathrm{AL}$ for attraction and projectile risk $(3000 \mu \mathrm{T})$ and the ELV for sensory effects $(2 \mathrm{~T})$ and health effects (8 T) set by Directive 2013/35/EU.

After measuring the magnetic flux densities, the measurements were compared to values calculated with Ampere's law, which relates the integrated magnetic field around a closed loop to the electric current passing through the loop, and can be written as shown in equation (1):

$$
\oint_{C} \mathbf{B} \cdot d \mathbf{l}=B * 2 \pi r=\mu_{0} I_{\mathrm{enc}} .
$$

Quantities in equation (1) are as follows: $B$ is the magnetic flux density, $r$ is the distance to the center line of a straight cable, $\mu_{0}$ is the permeability of free space and $I_{\text {enc }}$ is the loading current. Measured and calculated magnetic flux densities can be seen in Figures 4 and 5 .

Figures 4 and 5 show that the measured values vary approximately $30 \%$ from values calculated with Ampere's law. This is likely to result from error in estimated distances between the measurement probe and the cables, as distances were estimated ocularly. However, the measured values exceeded the calculated values of Ampere's law on FennoSkan 2 and therefore the measurements have a safety margin compared to theoretical values. On Fenno-Skan 1, the calculated values were higher than the measurements, and so it is safer to add a safety margin to the distances that indicate the safe range from Fenno-Skan 1.

\section{Discussion}

Measurements show that close to the Fenno-Skan 1 and Fenno-Skan 2 cable terminals, the AL for interference with active implanted devices $(500 \mu \mathrm{T})$ may be exceeded. This is dependent on the current of the cables and therefore varies from time to time. As the magnetic flux density is directly proportional to the current and the highest DC current flow in the Fenno-Skan 2 cable, it can be said that the highest static magnetic field in Fingrid's grid appears in the vicinity of the Fenno-Skan 2 cable, assuming it is operating at full capacity.

The measured results differed approximately 30\% from calculated values. Most of the uncertainty is likely caused by the inaccurate evaluation of the distances between the cables and the measurement probe. Also the used meter was calibrated two years before the measurements which can cause some error. In addition, the measurements should be made from multiple distances and with repetition. Nevertheless, the study gives a good approximation of static magnetic fields in Finland and can be used as a base for upcoming measurements and studies.

As workers might be exposed to static magnetic fields higher than the $500 \mu \mathrm{T}$ AL close to HVDC cables, and it might cause interference with active implanted devices, it is recommended that workers with medical devices do not take part in work tasks concerning live HVDC cables in Finland. However, by staying 

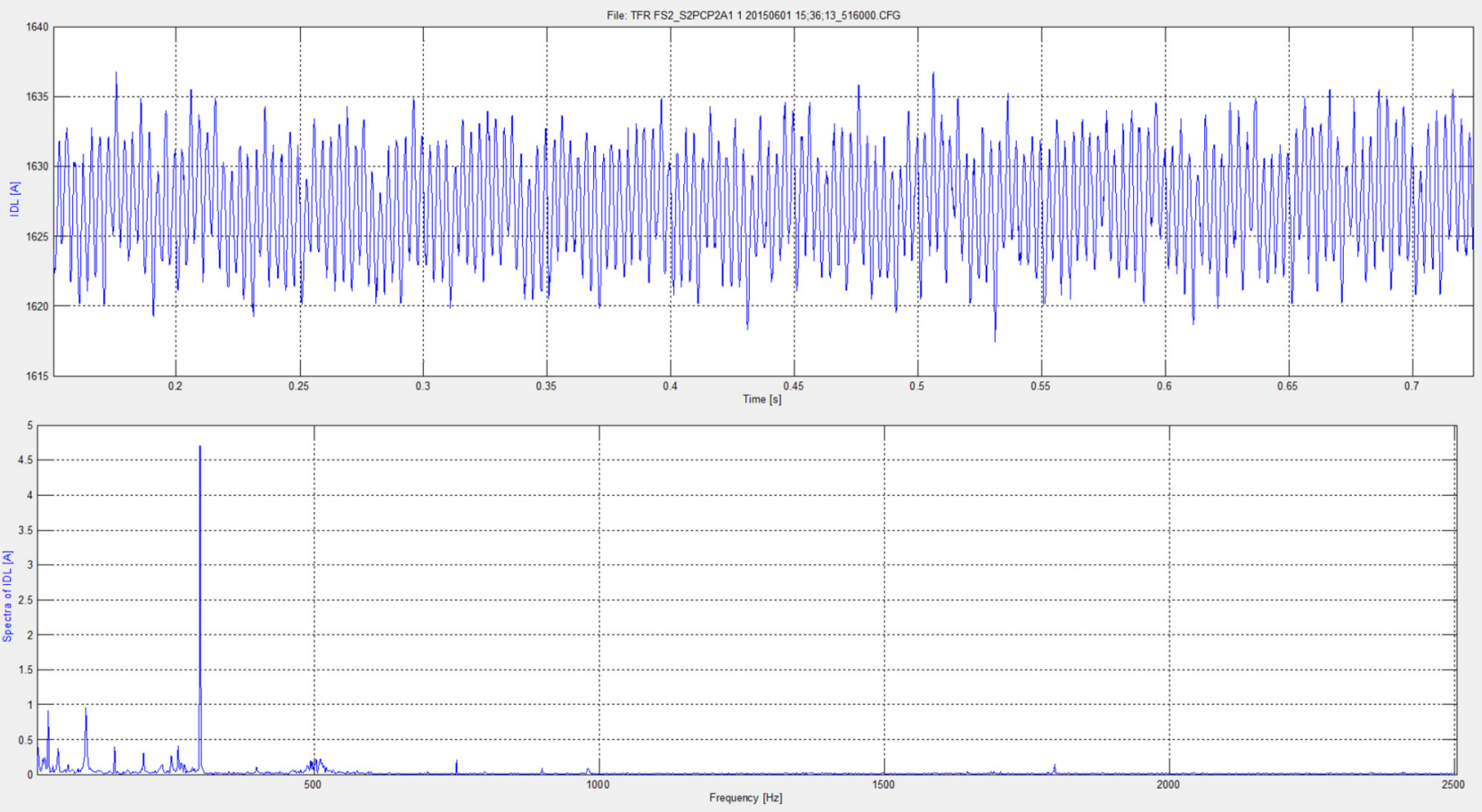

Fig. 1. Current and AC spectrum of Fenno-Skan 2 cable in normal operation measured at Rauma converter station. Upper measurement is the amplitude of the DC current in Amperes and the lower measurement is the AC spectrum in Amperes.
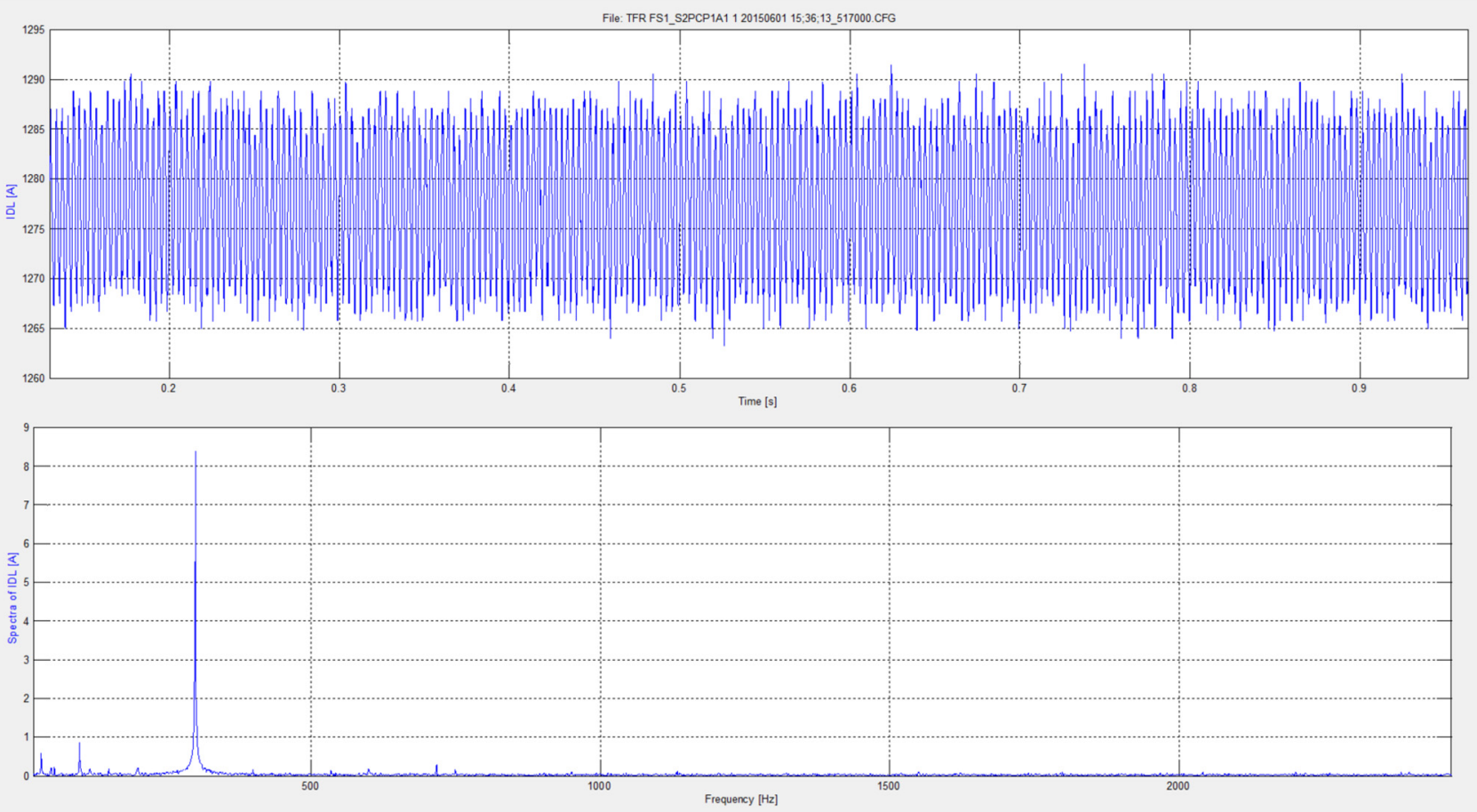

Fig. 2. Current and AC spectrum of Fenno-Skan 1 cable in normal operation measured at Rauma converter station. Upper measurement is the amplitude of the DC current in Amperes and the lower measurement is the AC spectrum in Amperes.

farther than approximately $1 \mathrm{~m}$ away from any HVDC cables in Finland, it seems to be a sufficient precaution to prevent the exposure of static magnetic fields higher than the $500 \mu \mathrm{T}$ AL.
Even the highest measured static magnetic flux density of $2.8 \mathrm{mT}$ is not anywhere near to the ELVs for sensory effects (2 T) and health effects (8 T) of the Directive 2013/35/EU. 


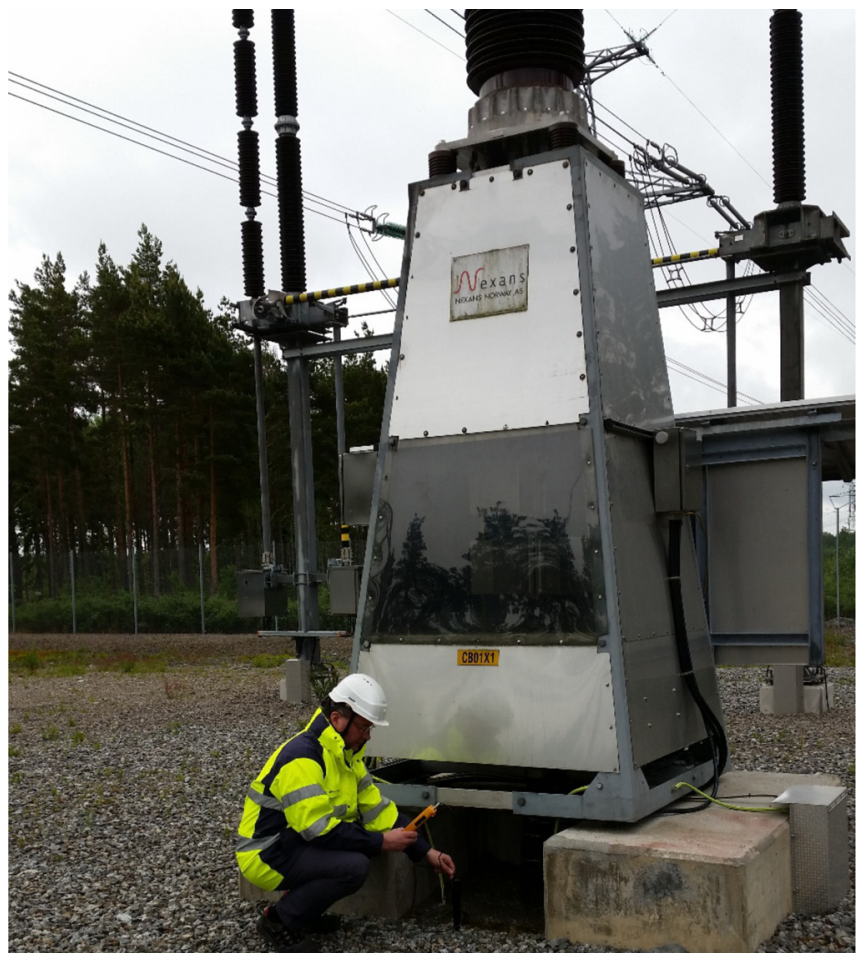

Fig. 3. Measuring static magnetic flux density at Fenno-Skan 2 cable terminal.

Table 1. Measurements of static magnetic flux densities. Distance from cable represents the distance between the center of the cable and the measurement probe.

\begin{tabular}{lll}
\hline Measurement site & $\begin{array}{l}\text { Distance } \\
\text { from cable }\end{array}$ & $\begin{array}{l}\text { Magnetic } \\
\text { flux density }\end{array}$ \\
\hline Fenno-Skan 2 & $0.1 \mathrm{~m}$ & $2800 \mu \mathrm{T}$ \\
Fenno-Skan 2 & $0.5 \mathrm{~m}$ & $900 \mu \mathrm{T}$ \\
Fenno-Skan 2 & $1.0 \mathrm{~m}$ & $500 \mu \mathrm{T}$ \\
Fenno-Skan 2 & $1.5 \mathrm{~m}$ & $300 \mu \mathrm{T}$ \\
Fenno-Skan 1 & $0.1 \mathrm{~m}$ & $1700 \mu \mathrm{T}$ \\
Fenno-Skan 1 & $0.5 \mathrm{~m}$ & $400 \mu \mathrm{T}$ \\
Fenno-Skan 1 & $1.0 \mathrm{~m}$ & $300 \mu \mathrm{T}$ \\
Underground & Ground level & $200 \mu \mathrm{T}$ \\
Fenno-Skan 2 & & \\
\hline
\end{tabular}

For comparison, the natural static magnetic field of the Earth is approximately $0.05 \mathrm{mT}$, which is $10 \%$ of the $500 \mu \mathrm{T}$ AL. The world's most powerful HVDC link, the JinpingSunan ultra high voltage direct current (UHVDC) link, in East China has nominal power of $7200 \mathrm{MW}$ and nominal current of $9000 \mathrm{~A}$ (ABB, 2013). That gives, according to the Ampere's law, magnetic flux density of $3 \mathrm{mT}$ at the distance of $0.6 \mathrm{~m}$ from the cable.

The only peak in the frequency spectrum of Fenno-Skan cables was found at around $300 \mathrm{~Hz}$ and the amplitude was $8.5 \mathrm{~A}$ at highest. For $300 \mathrm{~Hz}$ frequency the Directive 2013/35/EU gives a lower AL of $1000 \mu \mathrm{T}$ as the highest measured static magnetic flux density was $2800 \mu \mathrm{T}$. However, $8.5 \mathrm{~A}$ is only $0.6 \%$ of the nominal DC current and therefore AC fields are only very

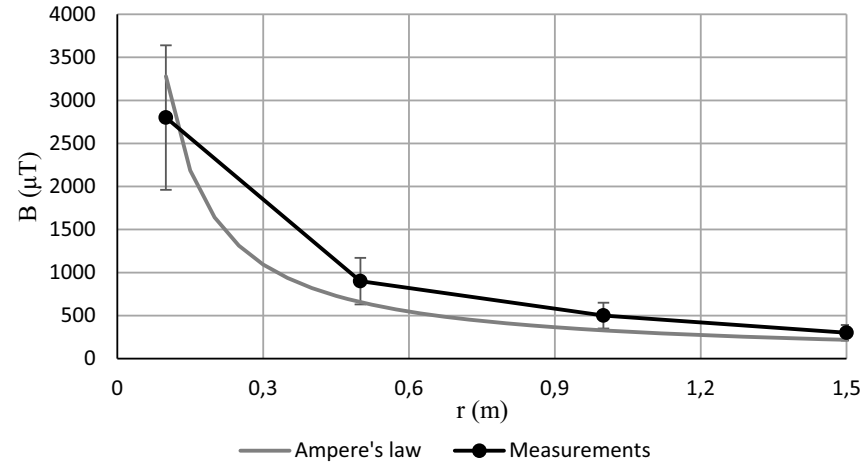

Fig. 4. Measured and calculated magnetic flux densities of FennoSkan 2 cable. The grey line represents the values calculated with Ampere's law, and the black line represents the measured values. The error marginals represent $30 \%$ range. The $x$-axis represents the distance from the cable in meters and $y$-axis the magnetic flux density in $\mu \mathrm{T}$.

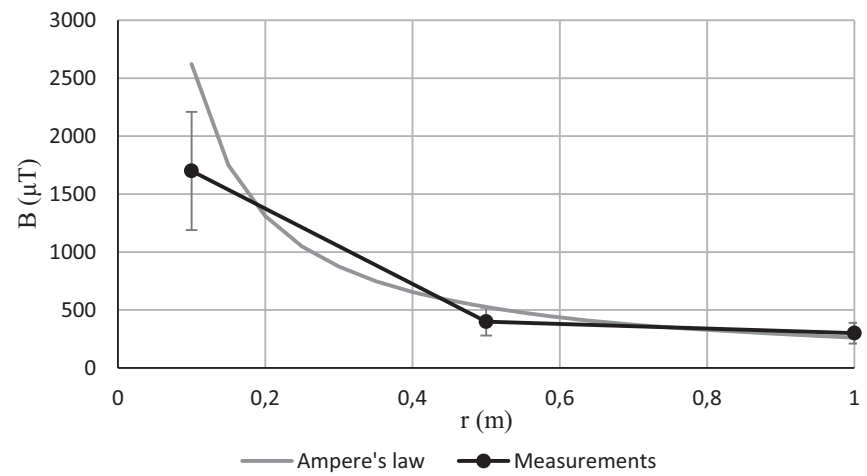

Fig. 5. Measured and calculated magnetic flux densities of FennoSkan 1 cable. The grey line represents the values calculated with Ampere's law, and the black line represents the measured values. The error marginals represent a $30 \%$ range. The $x$-axis represents the distance from the cable in meters and $y$-axis the magnetic flux density in $\mu \mathrm{T}$.

small fractions compared to static magnetic fields; AC fields are too low to cause significance to the study.

The calculations of the earlier Italian study are presented in magnetic field strengths. However, nowadays the exposure limits of magnetic fields are presented in magnetic flux densities. To be able to compare the magnetic field strengths and magnetic flux densities, we would need to know the exact permeability of the material between the measurement probe and the cable. An approximation could be done using the permeability of a vacuum, but in this case it do not give reasonable results, which indicates to incorrect permeability. Another difference between the measurements is the lower nominal current of the cable between Italy and France, which nevertheless can be taken into account.

However, in both studies the conclusions have been similar: the cables in question do not cause high enough static magnetic fields to prevent from working in the vicinity of the cables. Only exception is the workers with medical devices, who should not be working with HVDC cables, at least in Finland. Nevertheless, it is safe to use the approximations of this study as the reference while evaluating occupational 
exposure to static magnetic fields of cables with exact or lower currents than Fenno-Skan 2.

\section{Conclusion}

While conducting this study, it was found that the highest measured magnetic flux density was $2800 \mu \mathrm{T}$, which occurred inside the Fenno-Skan 2 cable terminal with the measurement probe attached to the cable. Therefore, all of the results were below the $3000 \mu \mathrm{T}$ high $\mathrm{AL}$ set by the Directive $2013 / 35 / \mathrm{EU}$, and only the $500 \mu \mathrm{T} \mathrm{AL}$ for interference with active implanted devices was exceeded. As all of the measurements were below the higher AL, it can be stated that the static magnetic fields occurring in Fingrid's grid are safe for workers who do not have active implanted devices.

\section{References}

ABB. 2013. Jinping-Sunan: the most powerful transmission line in the world. ABB-website: http://new.abb.com/systems/hvdc/refer ences/jinping — sunan (accessed 10.11.2016).

Bottauscio O, Chiampi M, Pastorelli M. 2009. Electromagnetic impact of underground HVDC cables. In: IEEE 13th European Conference on Power Electronics and Applications, 8-10 September 2009.
European Parliament and Council of the European Union. 2013. Directive 2013/35/EU of the European Parliament and of the council on the minimum health and safety requirements regarding the exposure of workers to the risks arising from physical agents (electromagnetic fields) (20th individual directive within the meaning of Article 16 (1) of directive 89/391/EEC) and repealing directive 2004/40/EC. Off. J. Eur. Union 21: 12-15.

Feychting M. 2005. Effects of static magnetic fields relevant to human health. Prog. Biophys. Mol. Biol. 87(2-3): 241-246.

Health Protection Agency (HPA). 2008. Static magnetic fields: report of the independent advisory group on non-ionising radiation. ISBN: 978-0-85951-616-7.

ICNIRP. 2009. Guidelines on limits of exposure to static magnetic fields. Health Phys. 96(4): 504-514.

Korpinen L, Pääkkönen R. 2010. Occupational exposure to electric and magnetic fields during work tasks at $110 \mathrm{kV}$ substations in the Tampere region. Bioelectromagnetics 31(3): 252-254.

Korpinen L, Kuisti H, Pääkkönen R, Vanhala P, Elovaara J. 2011. Occupational exposure to electric and magnetic fields while working at switching and transforming stations of $110 \mathrm{kV}$. Ann. Occup. Hyg. 55(5): 526-536.

Latva-Teikari J, Karjanlahti T, Kurikka-Oja J, Elovaara J, Långsjö T, Korpinen L. 2008. Measuring occupational exposure to electric and magnetic fields at $400 \mathrm{kV}$ substations. In: Transmission and Distribution Conference and Exposition, IEEE PES Powering Toward the Future, 4, Chicago, United States, 21-24 April 2008.

Cite this article as: Tonteri J, Heiskanen T, Elovaara J, Penttilä M, Korpinen L. 2017. Preliminary results of measuring occupational exposure to static magnetic fields at HVDC cables in Finland. Radioprotection 52(2): 119-123 